\title{
Movable Au Microplates as Fluorescence Enhancing Substrates for Live Cells
}

\author{
Boya Radha ${ }^{1}$, Mohammed Arif ${ }^{2}$, Ranjan Datta ${ }^{3}$, Tapas K. Kundu ${ }^{2}$, and Giridhar U. Kulkarni ${ }^{1}(\bowtie)$ \\ ${ }^{1}$ Chemistry and Physics of Materials Unit and DST Unit on Nanoscience, Jawaharlal Nehru Centre for Advanced Scientific Research, \\ Jakkur P.O., Bangalore 560064, India \\ ${ }^{2}$ Molecular Biology and Genetics Unit, Jawaharlal Nehru Centre for Advanced Scientific Research, Jakkur P.O., Bangalore 560064, India \\ ${ }^{3}$ International Centre for Materials Science, Jawaharlal Nehru Centre for Advanced Scientific Research, Jakkur P.O., Bangalore 560064, India \\ Received: 14 July 2010 / Revised: 24 August 2010 / Accepted: 31 August 2010 \\ C The Author(s) 2010. This article is published with open access at Springerlink.com
}

\begin{abstract}
Hexagonal and triangular Au microplates extending over an area of $\sim 12,000 \mu \mathrm{m}^{2}$ with thickness in the range 30-1000 $\mathrm{nm}$ have been synthesized using a single step thermolysis of $\left(\mathrm{AuCl}_{4}\right)^{-}$-tetraoctylammonium bromide complex in air. The microplates are self-supporting and can be easily manipulated using a sharp pin, a property which enables them to serve as substrates for living cells. The microplate surface is non-toxic to living cells and can enhance the fluorescence signal from fluorophores residing within the cell by an order of magnitude. In addition, the microplates are smooth and single-crystalline, and ideal as microscopy substrates and molecular electrodes. The growth of the microplates in the initial stages is interesting in that they seem to grow perpendicular to the substrate, as evidenced by in situ microscopy.
\end{abstract}

\section{KEYWORDS}

Au microplates, synthesis, cell substrate, metal enhanced fluorescence, manipulation

\section{Introduction}

Anisotropic gold nanostructures have attracted a great deal of attention in recent years due to shape-induced properties of interest in catalytic, electrical and optical applications [1-3]. As a triumph of bottom-up approaches using chemical methods, there is much emphasis on growing polygonal plate-like structures $[4,5]$ with projected applications as infrared absorbing optical coatings [6], surface plasmon resonators [7], high activity electrocatalysts [8], STM substrates [9], and as substrates for surface enhanced Raman spectroscopy [10]. For such applications it is desirable to have plates as large as possible, but of submicron thickness, without compromising with the flatness and surface smoothness. Towards this end, there have been close to 100 reports of such gold materials during the last decade (see Table S-1 in the Electronic Supplementary Material (ESM)). Most of them report sub-micron and few-micron sized Au hexagonal and triangular plates, and wider plates with sides extending over tens of microns are not commonly found; in the reported instances [11-14], the plates seem fragile, contain defects, and are mingled with other nanostructures. The separation of the desired plates from the mass is like removing a needle from haystack! Thus, the manipulation and organization of the as-made plates has proved rather difficult. In one recent instance, the

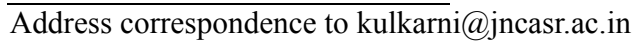


separation and assembly of small gold triangles was made possible by selective precipitation [15]. Topdown methods too, have aimed at producing faceted structures for use as a substrate in scanning tunneling microscope (STM) studies [16]. Generally, these structures have unfriendly geometries and macroscopic thickness, making them unsuitable for many applications. Thus, it has not been trivial to produce metal microplates with the requisite qualities. Here, we report a simple method involving thermolysis of a gold-organic complex in air, which affords unusually large $(>150 \mu \mathrm{m})$ hexagonal, triangular and related polygonal $\mathrm{Au}$ microplates on flat substrates such as glass. We have monitored the growth of the particles in situ and examined their crystallinity using high resolution electron microscopy (HRTEM). We also demonstrate their utility in fluorescence enhancing platforms for living cells.

\section{Results and discussion}

The precursor was $\left(\mathrm{AuCl}_{4}\right)^{-}$ions stabilized by tetraoctylammonium bromide in toluene [17] (25 mmol/L), denoted $\mathrm{Au}-\mathrm{ToABr}$. The synthesis process involved two steps, drop coating or spin coating of $\mathrm{Au}-\mathrm{ToABr}$ precursor onto a given substrate and subsequent thermolysis at $130{ }^{\circ} \mathrm{C}$ in air to give reduced $\mathrm{Au}$ (see the scheme in Fig. 1(a)). After thermolysis, the unreacted $\mathrm{Au}-\mathrm{ToABr}$ was washed off with toluene. The substrate can be $\mathrm{Si}$, glass, stainless steel, polyimide, polydimethylsiloxane (PDMS), mica or highly oriented pyrolytic graphite (HOPG) - almost anything that can withstand the temperature and remain insoluble in the solvent. After thermolysis for $24 \mathrm{~h}$, the reduced gold was in the form of microplates, with many of them having edge lengths above $100 \mu \mathrm{m}$. The Au microplates are visible even to the unaided eye. The optical microscopy images of a few typical plates are shown in Fig. 1(b). As shown in the histogram in Fig. 1(c), microplates with areas of $5000-10,000 \mu \mathrm{m}^{2}$ were commonly seen. The thickness was most commonly sub-100 nm (Fig. 1(d)). The area and thickness had no definite relation: in the two cases shown in Fig. 1(e), the smaller hexagon (top) is $\sim 1 \mu \mathrm{m}$ thick while the thickness of the bottom truncated triangle is only $\sim 100 \mathrm{~nm}$. Figure 1(f) shows a typical low magnification scanning electron microscopy
(SEM) image with several microplates covering the substrate; there are very few particulates. Importantly, they maintain smoothness $(\sim 1 \mathrm{~nm})$ even over large areas as revealed by optical profilometry (Fig. S-1 in the ESM). The smoothness should render them suitable for microscopy studies. Moreover because of their large size, they are easily identifiable without the aid of markers. Some control over size distribution is possible. A higher synthesis temperature $\left(250{ }^{\circ} \mathrm{C}\right)$ was found to narrow down the distribution, but the mean size was also reduced (Fig. S-2 in the ESM). Another attractive feature is that the plates can be detached from the substrate rather easily. A sharp pin carrying an $\mathrm{Au}$ microplate is shown in Fig. 2(a). Shown in Fig. 2(b) is a pattern of the microplates organized using the sharp pin. The transferred microplates are quite mobile on a PDMS surface, requiring little force (Fig. S-3 in the $\mathrm{ESM}$ ). In one report, manipulation of a nanowheel made from a Au nanoplate was shown using a nanomanipulator inside a focused ion beam (FIB) system [18]. In the present case, the process is greatly simplified by virtue of the large size of the microplates, such that no sophisticated instrumentation is required for manipulation. These microplates can also be dropped into microwells as shown in Fig. 2(c).

The surface of the microplates is devoid of possible contaminants such as carbon as revealed by energy dispersive spectroscopy (EDS) mapping data (Fig. S-4 in the ESM). X-ray photoelectron spectra in the C 1s core-level region $(284 \mathrm{eV})$ had negligible intensity. The $\mathrm{Au} 4 \mathrm{f}_{7 / 2}$ core level spectrum was intense, with the peak position at $\sim 84 \mathrm{eV}$ being typical of metallic $\mathrm{Au}$. Figure 3(a) shows transmission electron microscopy (TEM) image of a truncated triangular plate. The contours seen in the image originate from the diffraction effects arising due to slight bending of the thin plate on the TEM grid surface [19]. The selected area electron diffraction (SAED) pattern shown in the inset is hexagonally symmetric and corresponds to the facecentered cubic (FCC) Au crystal lattice viewed along the $\langle 111\rangle$ direction. The spots representing $\{220\},\{422\}$, and $\{440\}$ Bragg planes are clearly visible. Importantly, we do not see any kinematically forbidden spots such as $1 / 3\{422\}$, which have been attributed in other systems to the presence of two or more twin planes in the nanoplates $[19,20]$. The HRTEM image along the 


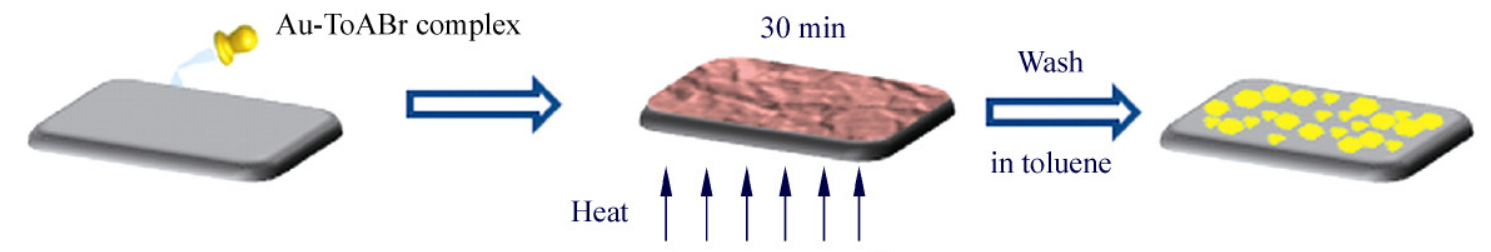

(a)

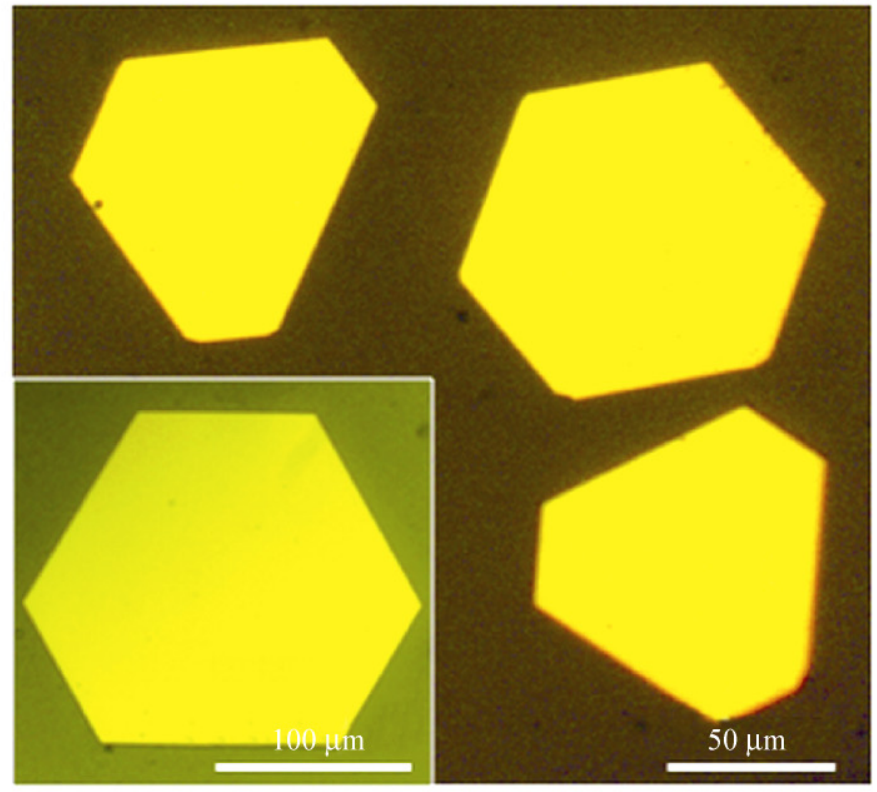

(b)

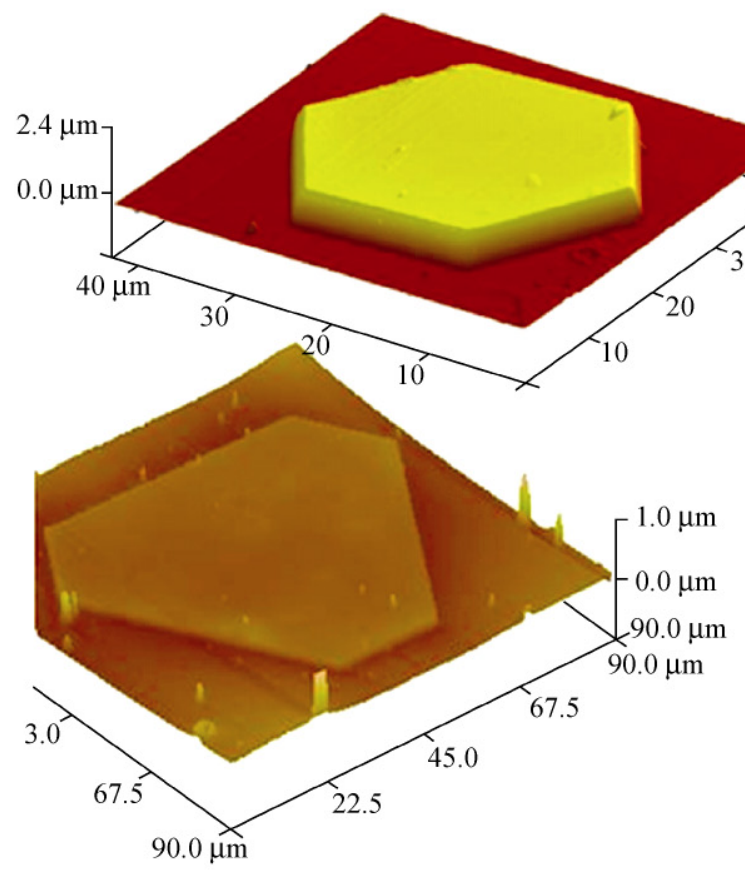

(e)

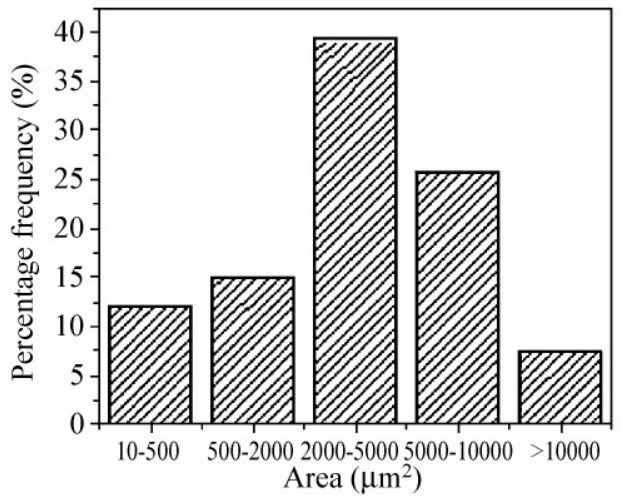

(c)

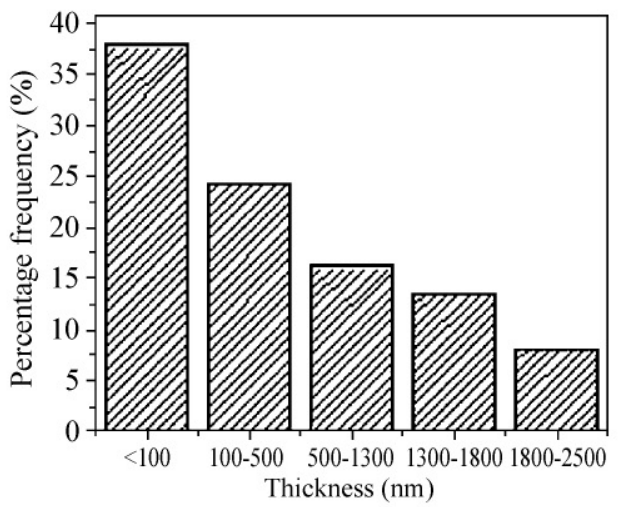

(d)

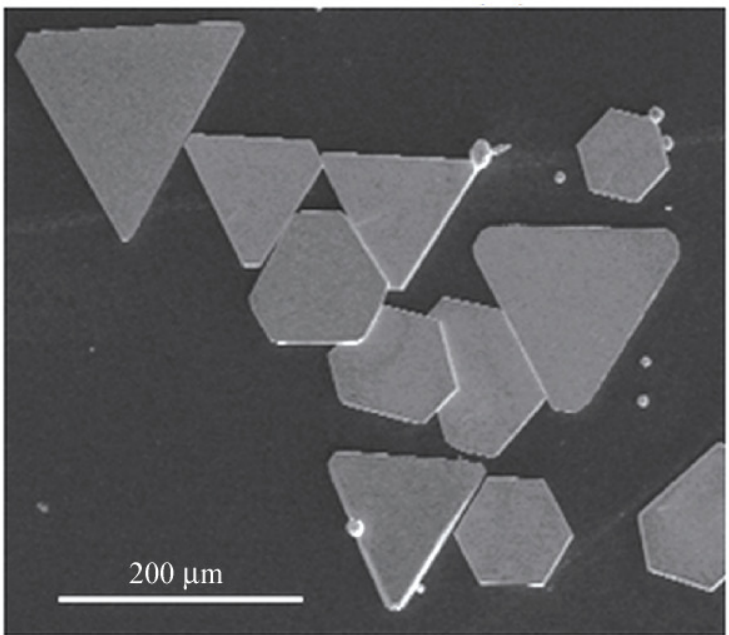

(f)

Figure 1 (a) Schematic illustration of the procedure for making Au microplates. First, Au-ToABr solution is coated onto the desired substrate and then thermolysed at $130{ }^{\circ} \mathrm{C}$ for $6 \mathrm{~h}$. (b) Optical image showing the microplates, with a large one with an area of $\sim 12,000 \mu \mathrm{m}^{2}$ shown in the inset. Histograms of (c) the area of the plates and (d) the thickness of the plates. (e) AFM topography images of a thick microplate (top) and a thin one (bottom). (f) A low magnification SEM image showing several Au microplates on the substrate 


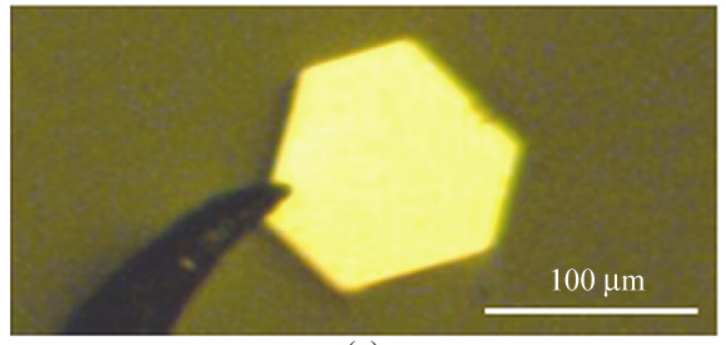

(a)

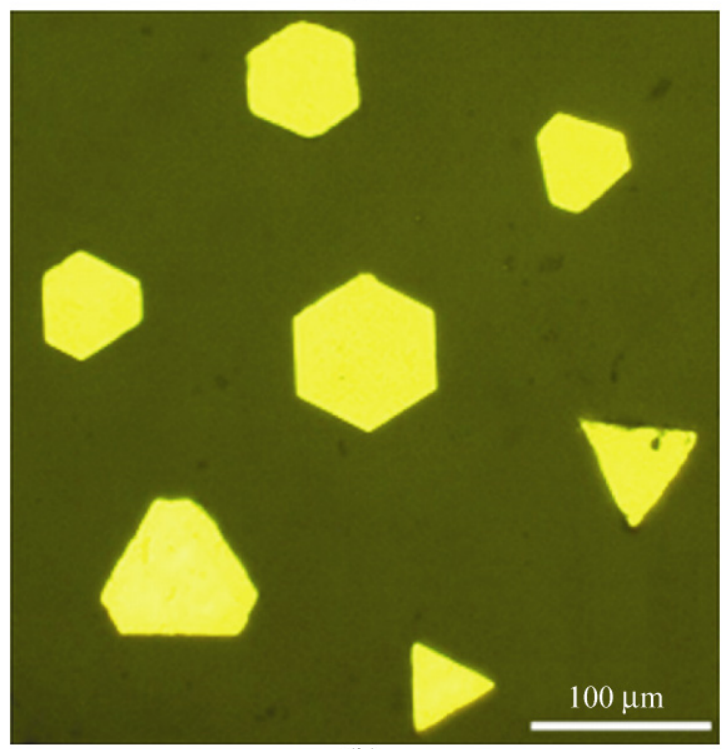

(b)

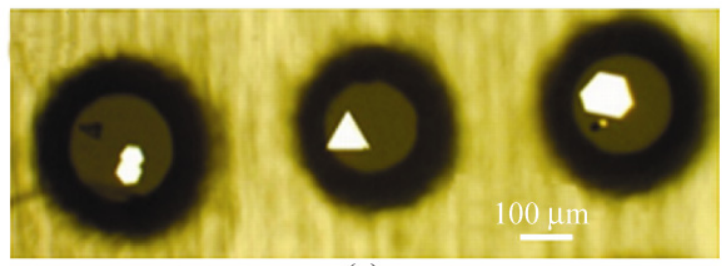

(c)

Figure 2 (a) Optical image of a sharp pin carrying a Au microplate. (b) Au microplates arranged in a pattern on a PDMS substate. For this purpose, the as-made $\mathrm{Au}$ microplates were dispersed in toluene, and then a drop of toluene containing Au microplates was placed on a PDMS surface. The plates were manipulated using a pin. (c) Microplates placed in microwells formed between a stencil and a glass substrate. A drop of toluene containing Au microplates was spread on a stencil. As the solvent evaporated, Au microplates became arranged in the wells, one in each, presumably because the size of the wells was chosen to be similar to that of the microplates

$\langle 111\rangle$ zone axis in Fig. 3(b) reveals Au atoms (white dots) in hexagonal packing with a spacing of $\sim 1.4 \AA$ between the rows, which corresponds to the $\{220\}$ planes. This observation is consistent with the geometric model of an FCC crystal, when viewed along the $\langle 111\rangle$ direction. The overall crystallinity of the microplates distributed on a substrate is further supported by the X-ray diffraction (XRD) data, which showed preferential (111) orientation (Fig. S-5 in the ESM). The relative diffraction intensity of the (200) reflection with respect to (111) is found to be 0.02 . We believe that this is the lowest value obtained in the literature for such microplates (Fig. S-5 in ESM).

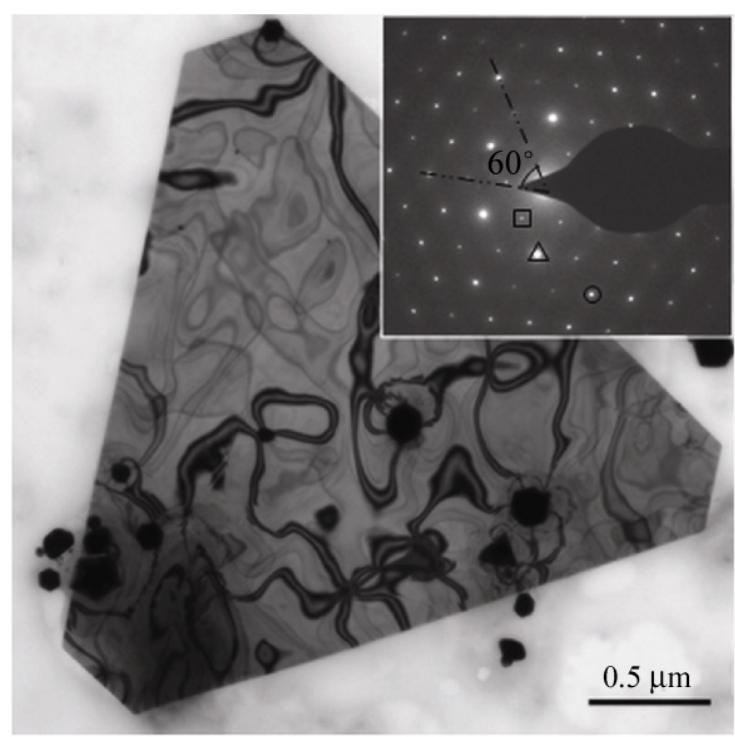

(a)

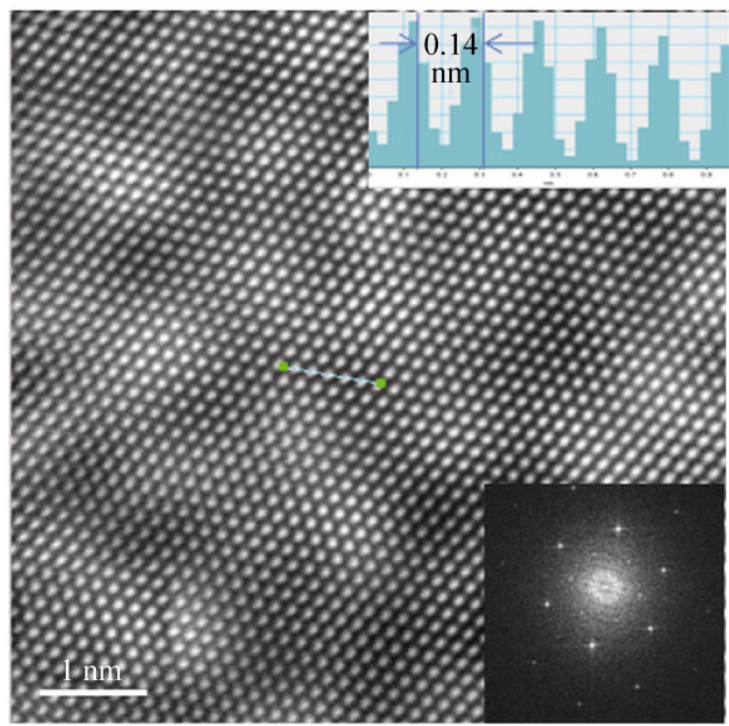

(b)

Figure 3 (a) TEM image of the Au microplate, with the SAED pattern shown as an inset. The SAED is hexagonally symmetric and the spots marked by a square, triangle, and circle correspond to $\{220\},\{422\}$, and $\{440\}$ planes, respectively. (b) HRTEM image of the microplate; the corresponding fast Fourier transform (FFT) is shown as inset. The bright dots are Au atoms, with the spacing marked in the inset 
We have monitored the growth of $\mathrm{Au}$ microplates at two different length scales using SEM and optical microscopy (Fig. 4). After thermolysis for just one minute, the resulting $\mathrm{Au}$ nanoparticles are arranged in the form of very tiny $(<100 \mathrm{~nm})$ triangle shaped plates, as shown in Fig. 4(a). The nucleation and growth of these embryonic structures take place in a confined boundary defined by the drop of the precursor (Fig. 4(b)). A halo around the growing structure is commonly seen (Fig. 4(c)), which may arise from the $\mathrm{Au}-\mathrm{ToABr}$ feedstock continuously supplying $\mathrm{Au}$ atoms. Under an optical microscope, we have seen something very interesting. As thermolysis of Au$\mathrm{ToABr}$ begins, a large number of tiny microplates are seen all over the substrate, often perpendicular to the substrate (Fig. S-6 in the ESM). As shown in Fig. 4(d), the triangular microplate appears as if one of the corners of the microplate is pinned to the substrate. The growth proceeds out from the free standing corners and, as a result the two corners seem to move away from each other (Movie S-1 in the ESM). After a certain amount of growth, the microplate falls flat (Fig. 4(e)) and continues to grow outward from the same corners. It now looks brighter as it lies flat on the substrate.
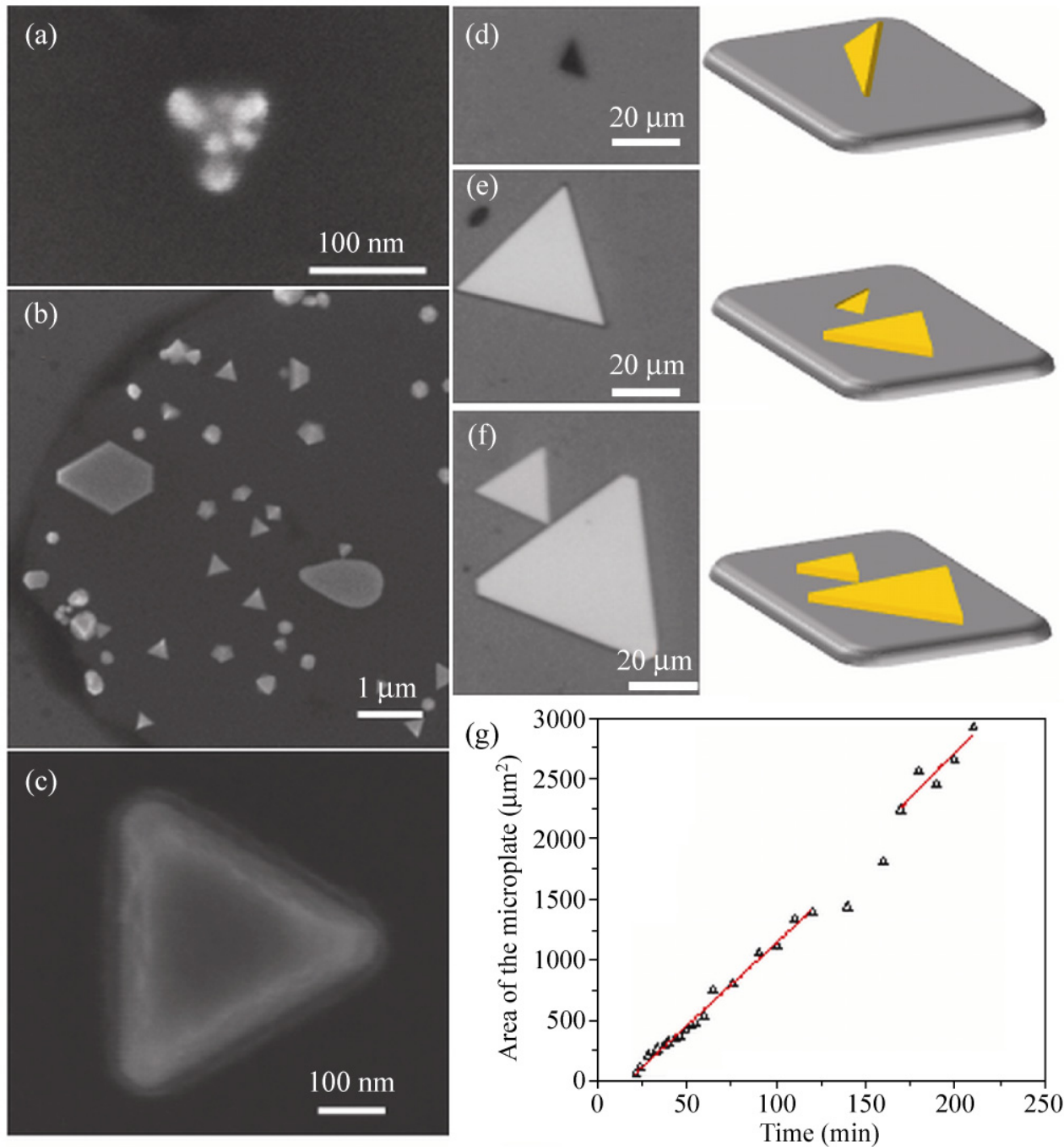

Figure 4 SEM images of the products formed after thermolysis of the Au-ToABr complex on a Si substrate for (a) 1 min, (b) 2 min, and (c) $5 \mathrm{~min}$ at $250{ }^{\circ} \mathrm{C}$. Optical images (d)-(f) of a triangular Au microplate as it grew, with schematic illustrations alongside. The darker shade of the microplate in (d) is due to its angle with respect to the optical axis. (g) Plot of the area of the triangular microplate versus the thermolysis time. A slight deviation from linearity is seen around $150 \mathrm{~min}$. This coincides with the beginning of truncation of the microplate corners as seen in (e) 
Another tiny microplate is seen to nucleate in the neighborhood. In such circumstances, the neighboring plates usually come closer and reorient as shown in Fig. 4(f) (also see Movie S-1 in the ESM). The synthesis of $\mathrm{Au}$ microplates using our method does not seem to involve the mediation of the solvent, as the minimum temperature required is greater than the boiling point of the solvent (toluene has a boiling point of $110^{\circ} \mathrm{C}$, whereas a thermolysis temperature of $130^{\circ} \mathrm{C}$ was employed). There is essentially a direct conversion of the metal precursor in the liquid-crystalline state to metallic Au. As seen in the dynamic optical microscopy images, the Au nucleus which forms the vertex of the growing microplate is surrounded by the liquid-crystalline precursor which serves as a feedstock for $\mathrm{Au}$. The viscous nature of the precursor does not seem to favor in-plane growth but rather forms a supportive matrix on either side of the growing plate. Around $60 \%$ to $70 \%$ of the plates tend to grow perpendicular to the substrate. Those which grow in-plane, are restricted in size. The crystal structure of the substrate surface has no direct bearing on the growth of the plates, although the surface morphology may influence the overall size. A rough substrate surface may also restrict steady supply of the precursor to the growing plate. As seen in Fig. 4(g), the area of the triangular plate increased linearly with the time of thermolysis, implying that the growth is diffusion limited [21]. The slope may depend on the local concentration of the precursor and the temperature, among other conditions. A slight deviation from linearity seen around $150 \mathrm{~min}$, is concomitant with the microplate corners becoming rounded and truncated (Fig. 4(e)).

The microplates presented above have some unique properties: they are large, flat, clean, and movable. These qualities prompted us to explore their utility as platforms for hosting living cells. Previously, Ishihara et al. [22] have fabricated mobile Parylene microplates which could host adherent cells. Transparent $\mathrm{SiO}_{2}$ microplates [23] have also been employed for the same purpose. Such surfaces generally require chemical modification by a plasma pretreatment before attaching the cell. There have been reports of cell patterning on $\mathrm{Au}$ substrates by surface engineering [24,25] with extracellular matrix proteins. The cellular behavior in terms of adaptation and signaling depends crucially on the environment. In the present study, we have used coverslips coated with $\mathrm{Au}$ microplates for growing three different cell lines-KB (human oral cancer), H1299 (human lung carcinoma) and 3T3 (mouse fibroblast) cells. The cells were seen attached to the microplates as well as on other regions of the coverslips (Fig. 5). Importantly, they were as healthy on the plates as on the coverslip surface itself, implying that there is no cellular toxicity arising from the microplates. Here, it should be stressed that no surface chemical modification was required in order to attach the cells. Electrostatic interactions between the cell membrane and the Au surface, as well as specific interactions involving amine groups in cell surface proteins, may be responsible for the attachment of cells to the microplates. Thus, the microplates provide an opportunity to carry out cellular studies, free of any of the effects which inevitably arise after surface modifications. Brunetti et al. [26] have recently shown the possible role of nanoroughness in determining the cytophilic or cytophobic nature of a surface for neuronal cells. The cells are directed preferentially towards the flat regions of the surface (cytophilic). Although the cell line used here is different, the surface smoothness of the microplate would be advantageous in terms of attracting live cells. The microplate is also seen to enhance the fluorescence of Hoechst 33528 dye in the nucleus, as shown in Figs. 5(a) and 5(b). This is akin to metal-enhanced fluorescence [27] (MEF) which increases the fluorescence emission from a fluorophore. MEF arises because of the increase in the electric field near the metal surface in the presence of the incident light [28]. It is being increasingly used for cell imaging as the chosen fluorophores may possess low quantum yields, making them difficult to visualize, particularly at lower concentrations [28]. As shown in Figs. 5(a) and 5(b), the cell situated in the middle of the plate is seen with a bright contrast while the cells around the plate resting on the coverglass are seen lightly colored. Comparing the relative sizes, it is clear that the microplate can essentially act as a single cell platform (Figs. S-7 and S-8 in the ESM). Another cell is placed half on the plate and half on the coverglass. Fluorescence enhancement by the microplate surface is clearly evident. The extent of enhancement is quantitatively expressed in Figs. 5(c) and 5(d). In some 

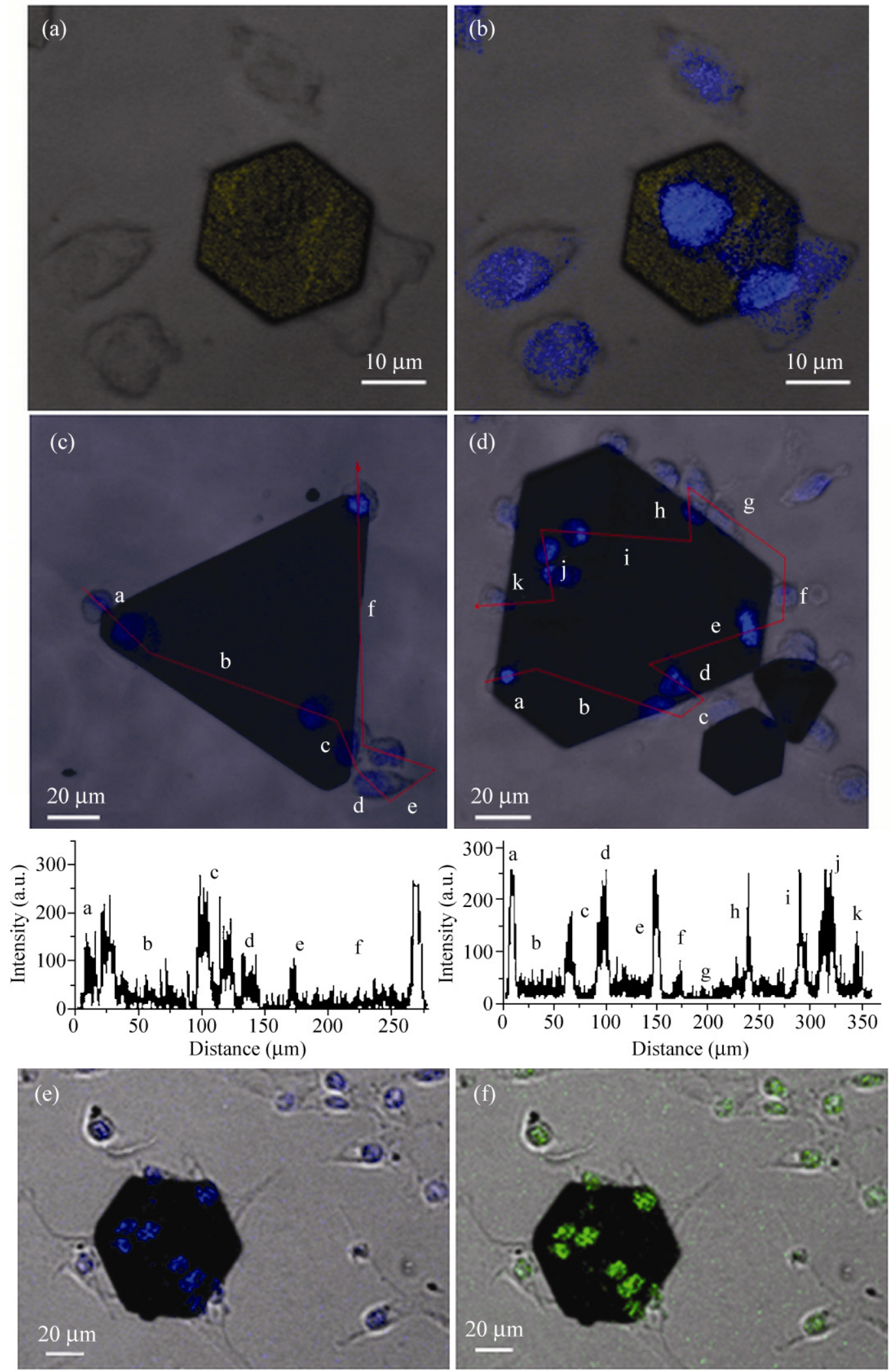

Figure 5 (a) and (b) Confocal images showing a Au microplate hosting H1299 cells in which chromosomal DNA in the nucleus has been stained by Hoechst 33528 dye. (c) and (d) Two microplates hosting KB cells with Hoechst 33528 staining. The fluorescence enhancement is shown quantitatively in the plots below, with points a-k being a guide to the trace. (e) and (f) Confocal images of a Au microplate hosting mouse 3 T3 cells, stained with Hoechst and Alexa 488 in order to visualize the nucleus and the acetylated histone H3, respectively 
cases, the enhancement is nearly 10 times relative to plain regions of the coverglass, with the average enhancement factor being 5. Another important observation is that the blue emission is from the intracellular parts. As Hoescht 33528 dye is known to stain specifically the chromosomal DNA in the nucleus, what we see in Figs. 5(b)-5(d) clearly refers to the cell nuclei, of course within the MEF range. The excitation of plasmon modes at the metal surface is known to yield intense optical fields at short distances from the metal surface (a few tens of nanometers, typically) which may not play a significant role in case of micrometer-sized samples [26]. In the context of this study, the microsized fluorophore object (part of the stained cell) is held beyond the distance relevant for surface plasmon effects (> $30 \mathrm{~nm}$, as the cell membrane is typically thicker than this), hence, only classical effects seem to influence the fluorescence. In a previous study where plasmonic structures were employed, little or no enhancement was observed from nuclear dyes [29]. Another noteworthy advantage is that the 'mirror-type' classical enhancement is applicable to all visible wavelengths, unlike plasmonic enhancement which is wavelengthdependent. Figures 5(e) and 5(f) illustrate 3T3 cells on the microplate, with the fluorescence enhancement evident for Hoechst 33528 and Alexa 488 dye. Further, we used another dye (Alexa 568)-conjugated secondary antibody to visualize tubulin protein in the cell. Importantly, the observed fluorescence signal was better from cells present on the Au plates (Fig. S-9 in the ESM).

\section{Methods}

\subsection{Synthesis and characterization}

The precursor, denoted $\mathrm{Au}-\mathrm{To} \mathrm{ABr}$, was prepared by phase transfer of $\mathrm{AuCl}_{4}^{-}$ions from an aqueous solution $(25 \mathrm{mmol} / \mathrm{L}, 3.2 \mathrm{~mL})$ to toluene $(8 \mathrm{~mL})$ using tetraoctylammonium bromide $(50 \mathrm{mmol} / \mathrm{L})$ as phase transfer agent. Since the first report [17] of its use in colloidal Au preparation, this complex is being widely used, together with a variety of reducing and stabilizing agents. The two-phase mixture was vigorously stirred until all the $\left(\mathrm{AuCl}_{4}\right)^{-}$ions were transferred into the organic layer. As the phase transfer proceeded, the yellow colored bottom aqueous layer became colorless and the top organic layer developed a red color. The organic layer was separated and drop coated onto the chosen substrate. Thermolysis was carried out in air at $130{ }^{\circ} \mathrm{C}$ for $1-12 \mathrm{~h}$. In our experience, the thermal treatment was more effective in producing microplates than using external reducing or stabilizing agents; all such attempts led to nanoparticle formation. Following thermolysis, a gentle wash in toluene was carried out to remove any undecomposed precursor. The resulting Au microplates were examined using an optical microscope (Laben, India) and a scanning electron microscope (Nova NanoSEM 600 instrument, FEI Co., The Netherlands). Atomic force microscopy (AFM) measurements were obtained using a Dimension 3100 scanning probe microscope (SPM) with a NanoScope IV controller (Veeco, USA) in the contact mode. Standard $\mathrm{Si}_{3} \mathrm{~N}_{4}$ cantilevers were used for the normal topography imaging. For TEM microscopy, microplates were dispersed in toluene and a drop was placed on a holey carbon grid. This grid was used for TEM and SAED analysis in a Titan ${ }^{3}$ transmission electron microscope with an accelerating voltage of $80-300 \mathrm{kV}$. High resolution TEM (HRTEM) image were recorded in an aberration corrected mode with a negative Cs of $\sim-30 \mu \mathrm{m}$ and a positive defocus $\Delta f$ of $\sim+8 \mathrm{~nm}$ (bright atom imaging contrast) [30]. Figure 2(b) displays the HRTEM image after Fourier filtering the underlying amorphous carbon specimen support film. PDMS used as a substrate for manipulating Au plates was prepared as follows: Sylgard 184 curing agent (Dow Corning) and its elastomer were mixed in the ratio 1:10 by mass. The mixture was then degassed under vacuum for $30 \mathrm{~min}$. PDMS was poured onto a polycarbonate flat surface and then cured in an oven at $70{ }^{\circ} \mathrm{C}$ overnight.

\subsection{Cell culture and immunofluorescence}

The KB or H1299 or 3T3 cells were grown continuously as a monolayer at $37{ }^{\circ} \mathrm{C}$ and $5 \% \mathrm{CO}_{2}$ in Dulbecco's Modified Eagle Medium (DMEM) (Sigma). The DMEM medium was supplemented with penicillin (100 units/mL), streptomycin $(100 \mathrm{mg} / \mathrm{mL})$, and $10 \%$ heat-inactivated fetal bovine calf serum (FBS) before use. To visualize the effect of gold microplates onto the cellular system KB, H1299 or 3T3, cells were cultured as a monolayer on cover slips (coated with

\section{Springer}


gold microplates) in DMEM medium. Cells were grown for $24 \mathrm{~h}$ and immunofluorescence was carried out as previously described [31]. Fixed cells were probed with anti-acetylated histone H3 (Calbiochem) polyclonal antibodies followed by secondary antibodies conjugated with Alexa 488 (Invitrogen). In the case of tubulin, fixed cells were probed with anti-tubulin monoclonal antibody (Calbiochem) followed by secondary antibodies conjugated with Alexa 568 (Invitrogen). Hoechst 33528 (Sigma) was used to stain the chromosomal DNA. The images were recorded using a Zeiss LSM 510 laser scanning confocal microscope.

\section{Conclusions}

We have made $\mathrm{Au}$ microplates using a single step thermolysis of a simple $\mathrm{Au}$-organic precursor. The microplates routinely bear edge lengths of $\sim 100-150 \mu \mathrm{m}$ amounting to area of $>10,000 \mu \mathrm{m}^{2}$, with the thickness being in the range $30 \mathrm{~nm}-1 \mu \mathrm{m}$. Interestingly, the plates seem to grow perpendicular to the substrate from a nucleation site, with the precursor feedstock supplying $\mathrm{Au}$ atoms from both sides. We have been able to capture images of their embryonic state, as well as of later stages as they grow in size into microplates. They are atomically smooth and highly crystalline with the $\mathrm{Au}(111)$ face forming the polygonal surface, a property which should qualify them as ideal microscopy substrates and electrodes in molecular electronics. We have found that the microplate surface is non-toxic to living cells and can enhance the fluorescence signal from a cell nucleus (Hoechst 33528 dye) by an order of magnitude. Importantly, the plates can be picked up using a pin and placed at a desired location and organized. This experimental platform hence offers exciting opportunities for single cell-based studies and in probing the complex and dynamic relationships between cells and their local environment. In addition, the above unique properties of the $\mathrm{Au}$ microplates should be of interest to researchers in molecular electronics and surface science.

\section{Acknowledgements}

The authors thank Professor C. N. R. Rao for his constant encouragement. Support from the Department of Science and Technology, India is gratefully acknowledged. The authors acknowledge Dr. Basavaraj, Veeco Lab, JNCASR, India for technical assistance. B. Radha thanks the Council of Scientific and Industrial Research, India for financial assistance.

Electronic Supplementary Material: Supplementary material (summary of the literature describing the synthesis of Au plate-like structures, optical profiler images, SEM images, optical images of the manipulation, energy dispersive spectroscopy (EDS) mapping, XRD, movie illustrating the growth of a $\mathrm{Au}$ triangular microplate, confocal images of Au microplates hosting single $3 \mathrm{~T} 3$ cells, $z$-slices and KB cells stained with Alexa 588 dye used to visualize tubulin) is available in the online version of this article at http://dx.doi.org/10.1007/s12274-010-0040-6 and is accessible free of charge.

Open Access: This article is distributed under the terms of the Creative Commons Attribution Noncommercial License which permits any noncommercial use, distribution, and reproduction in any medium, provided the original author(s) and source are credited.

\section{References}

[1] Eustis, S.; El-Sayed, M. A. Why gold nanoparticles are more precious than pretty gold: Noble metal surface plasmon resonance and its enhancement of the radiative and nonradiative properties of nanocrystals of different shapes. Chem. Soc. Rev. 2006, 35, 209-217.

[2] Tapan, K. S.; Andrey, L. R. Nonspherical noble metal nanoparticles: Colloid-chemical synthesis and morphology control. Adv. Mater.2010, 22, 1781-1804.

[3] Sajanlal, P. R.; Pradeep, T. Mesoflowers: A new class of highly efficient surface-enhanced Raman active and infraredabsorbing materials. Nano Res. 2009, 2, 306-320.

[4] Shankar, S. S.; Rai, A.; Ankamwar, B.; Singh, A.; Ahmad, A.; Sastry, M. Biological synthesis of triangular gold nanoprisms. Nat. Mater. 2004, 3, 482-488.

[5] Sun, X.; Dong, S.; Wang, E. Large-scale synthesis of micrometer-scale single-crystalline Au plates of nanometer thickness by a wet-chemical route. Angew. Chem. Int. Ed. 2004, 43, 6360-6363.

[6] Shankar, S. S.; Rai, A.; Ahmad, A.; Sastry, M. Controlling the optical properties of lemongrass extract synthesized gold nanotriangles and potential application in infrared-absorbing 
optical coatings. Chem. Mater. 2005, 17, 566-572.

[7] Wiley, B. J.; Lipomi, D. J.; Bao, J.; Capasso, F.; Whitesides, G. M. Fabrication of surface plasmon resonators by nanoskiving single-crystalline gold microplates. Nano Lett. 2008, 8, 3023-3028.

[8] Li, W.; Ma, H.; Zhang, J.; Liu, X.; Feng, X. Fabrication of gold nanoprism thin films and their applications in designing high activity electrocatalysts. J. Phys. Chem. C 2009, 113, 1738-1745.

[9] Dahanayaka, D. H.; Wang, J. X.; Hossain, S.; Bumm, L. A. Optically transparent $\mathrm{Au}\{111\}$ substrates: Flat gold nanoparticle platforms for high-resolution scanning tunneling microscopy. J. Am. Chem. Soc. 2006, 128, 6052-6053.

[10] Sabur, A.; Havel, M.; Gogotsi, Y. SERS intensity optimization by controlling the size and shape of faceted gold nanoparticles. J. Raman Spec. 2008, 39, 61-67.

[11] Li, C. C.; Cai, W. P.; Cao, B. Q.; Sun, F. Q.; Li, Y.; Kan, C. X.; Zhang, L. D. Mass synthesis of large, single-crystal Au nanosheets based on a polyol process. Adv. Funct. Mater. 2006, 16, 83-90.

[12] Zhu, J.; Shen, Y.; Xie, A.; Qiu, L.; Zhang, Q.; Zhang, S. Photoinduced synthesis of anisotropic gold nanoparticles in room-temperature ionic liquid. J. Phys. Chem. C 2007, 111, 7629-7633.

[13] Li, Z.; Liu, Z.; Zhang, J.; Han, B.; Du, J.; Gao, Y.; Jiang, T. Synthesis of single-crystal gold nanosheets of large size in ionic liquids. J. Phys. Chem. B 2005, 109, 14445-14448.

[14] Kawasaki, H.; Yonezawa, T.; Nishimura, K.; Arakawa, R. Fabrication of submillimeter-sized gold plates from thermal decomposition of $\mathrm{HAuCl}_{4}$ in two-component ionic liquids. Chem. Lett. 2007, 36, 1038-1039.

[15] Ha, T. H.; Kim, Y. J.; Park, S. H. Complete separation of triangular gold nanoplates through selective precipitation under CTAB micelles in aqueous solution. Chem Comm. 2010, 46, 3164-3166.

[16] Voigtlander, B.; Linke, U.; Stollwerk, H.; Brona, J. Preparation of bead metal single crystals by electron beam heating. $J$. Vac. Sci. Tech. 2005, 23, 1535-1537.

[17] Brust, M.; Walker, M.; Bethell, D.; Schiffrin, D. J.; Whyman, R. Synthesis of thiol-derivatised gold nanoparticles in a two-phase liquid-liquid system. J. Chem. Soc., Chem. Commun. 1994, 801-802.

[18] Yun, Y. J.; Park, G.; Ah, C. S.; Park, H. J.; Yun, W. S.; Ha, D. H. Fabrication of versatile nanocomponents using singlecrystalline Au nanoplates. Appl. Phys. Lett. 2005, 87, 233110.

[19] Bai, X.; Zheng, L.; Li, N.; Dong, B.; Liu, H. Synthesis and characterization of microscale gold nanoplates using Langmuir monolayers of long-chain ionic liquid. Cryst. Growth Des. 2008, 8, 3840-3846.

[20] Xie, J.; Lee, J. Y.; Wang, D. I. C. Synthesis of singlecrystalline gold nanoplates in aqueous solutions through biomineralization by serum albumin protein. J. Phys. Chem. C 2007, 111, 10226-10232.

[21] Yukselici, M. H. Growth kinetics of CdSe nanoparticles in glass. J. Phys.—Condens. Mat. 2002, 14, 1153-1162.

[22] Ishihara, H.; Kuribayashi, K.; Takeuchi, S. Arraying single adherent cells by microplate self-assembly. In Micro Electro Mechanical Systems. IEEE 22nd International Conference on MEMS, 25-29 Jan. 2009, pp. 367-370.

[23] Onoe, H.; Takeuchi, S. Microfabricated mobile microplates for handling single adherent cells. J. Micromech. Microeng. 2008, 18, 095003.

[24] Veiseh, M.; Wickes, B. T.; Castner, D. G.; Zhang, M. Guided cell patterning on gold-silicon dioxide substrates by surface molecular engineering. Biomaterials 2004, 25, 3315-3324.

[25] Yousaf, M. N.; Houseman, B. T.; Mrksich, M. Using electroactive substrates to pattern the attachment of two different cell populations. Proc. Natl. Acad. Sci. USA 2001, 98, 5992-5996.

[26] Brunetti, V.; Maiorano, G.; Rizzello, L.; Sorce, B.; Sabella, S.; Cingolani, R.; Pompa, P. P. Neurons sense nanoscale roughness with nanometer sensitivity. Proc. Natl. Acad. Sci. USA 2010, 107, 6264-6269.

[27] Moal, E. L.; Fort, E.; Levque-Fort, S.; Cordelières, F. P.; Fontaine-Aupart, M. P.; Ricolleau, C. Enhanced fluorescence cell imaging with metal-coated slides. Biophys. J. 2007, 92, 2150-2161.

[28] Aslan, K.; Gryczynski, I.; Malicka, J.; Matveeva, E.; Lakowicz, J. R.; Geddes, C. D. Metal-enhanced fluorescence: An emerging tool in biotechnology. Curr. Opin. Biotech. 2005, 16, 55-62.

[29] Zhang, J.; Fu, Y.; Liang, D.; Zhao, R. Y.; Lakowicz, J. R. Enhanced fluorescence images for labeled cells on silver island films. Langmuir 2008, 24, 12452-12457.

[30] Jia, C. L.; Lentzen, M.; Urban, K. Atomic-resolution imaging of oxygen in perovskite ceramics. Science 2003, 299, 870-873.

[31] Selvi, B. R.; Jagadeesan, D.; Suma, B. S.; Nagashankar, G.; Arif, M.; Balasubramanyam, K.; Eswaramoorthy, M.; Kundu, T. K. Intrinsically fluorescent carbon nanospheres as a nuclear targeting vector: Delivery of membrane-impermeable molecule to modulate gene expression in vivo. Nano Lett. 2008, 8, 3182-3188. 